\title{
Dynamical gap driven by Yukawa coupling in holography
}

\author{
Jian-Pin $\mathbf{W u}^{1,2, a}$ \\ ${ }^{1}$ Center for Gravitation and Cosmology, College of Physical Science and Technology, Yangzhou University, Yangzhou 225009, China \\ ${ }^{2}$ School of Aeronautics and Astronautics, Shanghai Jiao Tong University, Shanghai 200240, China
}

Received: 19 August 2018 / Accepted: 6 August 2019 / Published online: 19 August 2019

(C) The Author(s) 2019

\begin{abstract}
We provide a novel mechanism to model the emergence of the dynamical gap in holography. It is implemented by introducing a novel coupling term, Yukawa interaction between the scalar field and the spinor field, in the Dirac action. By studying the fermionic spectral function, we find that the Yukawa coupling drives a dynamical gap formation. Moreover, the Yukawa coupling results in rearrangements of the spectrum at low frequency region but the density of states (DOS) for all Yukawa coupling parameter tend to the same value at the high frequency region, which is different from the well studied case with dipole coupling. We also study the evolution of the spectral function with temperature. We find that with the increase of temperature, the gap closes.
\end{abstract}

\section{Introduction}

Dynamical generation of a mass gap is a typical characteristic of many strongly coupling systems, for example, the 2 dimensional quantum chromodynamics [1] and the Mott insulator [2]. Since the latter is a strongly correlated electron system, it is hard to deal with these problems by the conventional methods. Instead, AdS/CFT correspondence [3-6] may provide understanding into the associated mechanisms of these systems by constructing a simple gravitational dual model.

In holographic framework, a hard gap in optical conductivity can be observed in [7]. This picture is implemented by axionic fields $\chi_{i}=k x_{i}$, which result in the momentum relaxation, in some gapped geometries as [7-10]. Further, some Mott-like insulator are proposed in [11-14]. In particular, a hard gap in insulating phase and Mott thought experiment can be implemented in [11,12].

The emergence of the dynamical gap has also been widely studied in holographic fermionic systems. The pioneer work appears in $[15,16]$. In this work, the chiral symmetrybreaking Pauli dipole coupling is introduced in the Dirac action. They study the fermionic response on top of ReissnerNordström-AdS (RN-AdS) background and observe the dynamical formation of Mott gap. This model exhibits two important characteristics of doped Mott insulator. One is the dynamical generation of a gap and another is the spectral weight transfer. The two features are robust for the holographic dipole coupling fermionic systems, which have been widely studied over more general geometries in [17-33]. In addition, in [34], they realize the holographic Fermi arcs by a chiral symmetry-preserving interaction, which is a modification of the chiral symmetry-breaking Pauli coupling studied in $[15,16]$.

In the presence of superconductivity, the gap can also come into being in the holographic fermionic spectrum. In holographic s-wave superconductor, the gap can be realized by a Majorana coupling [35]. Introducing spinor doublet coupling $S U$ (2) gauge field, the Fermi arcs can be implemented in holographic p-wave superconductor [36]. Adding the same Majorana coupling as [35] over holographic d-wave superconductor model, Fermi arcs and d-wave gap can also be generated [37].

In this paper, we propose a novel fermionic coupling term, Yukawa interactions, into the Dirac action and study its response. We observe the formation of the dynamical gap, which is driven by Yukawa coupling. This plan of this work is as follows. In Sect. 2, we introduce a non-trivial scalar field over RN-AdS black hole, which provides the Yukawa coupling between the spinor and the scalar field. And then, we introduce the spinor action with Yukawa coupling in Sect. 3 and derive the equation of motion (EOM) for the spinor field. The numerical results are presented in Sect. 4. In Sect. 5, we present the conclusion and discussion.

a e-mail: jianpinwu@yzu.edu.cn 


\section{Holographic framework}

In this section, following [38], we introduce a non-trivial scalar field profile, which provides a key ingredient to couple the spinor by Yukawa interaction. We describe it as follows. The action we consider reads [38]

$S=\int \mathrm{d}^{4} x \sqrt{-g}\left(R-Z(\Phi) \frac{L^{2}}{g_{F}^{2}} F^{2}-\frac{1}{2}\left(\nabla_{\mu} \Phi\right)^{2}-V(\Phi)\right)$,

with $Z$ and $V$ are given in what follows,

$V(\Phi)=-\frac{6}{L^{2}}+\frac{m^{2}}{2} \Phi^{2}$,

$Z(\Phi)=1+\alpha \Phi^{2}$

The mass of the scalar field relates its dimension as $m^{2} L^{2}=$ $\Delta(\Delta-3) . F=d A$ is the Maxwell field strength of $U(1)$ gauge field $A$. In what follows, for convenience, we set $g_{F}=$ 2 and $L=1$. And then, the equations of motion (EOMs) can be derived from the above action as

$R_{\mu \nu}-\left(3+\frac{1}{2} R\right) g_{\mu \nu}+\frac{1}{2}\left(T_{\mu \nu}^{A}+T_{\mu \nu}^{\Phi}\right)=0$,

$\nabla^{\mu}\left[\left(1+\alpha \Phi^{2}\right) F_{\mu \nu}\right]=0$,

$\left(\nabla^{2}-m^{2}-\frac{1}{2} \alpha F^{2}\right) \Phi=0$.

where

$T_{\mu \nu}^{A}=\left(1+\alpha \Phi^{2}\right)\left(\frac{1}{4} g_{\mu \nu} F^{2}-F_{\mu \rho} F_{\nu}{ }^{\rho}\right)$,

$T_{\mu \nu}^{\Phi}=-\nabla_{\mu} \Phi \nabla_{\nu} \Phi+\frac{1}{2} g_{\mu \nu}\left[\left(\nabla_{\mu} \Phi\right)^{2}+m^{2} \Phi^{2}\right]$.

We shall numerically construct the black brane solution with non-trivial scalar profile. We assume the following ansatz

$d s^{2}=\frac{1}{u^{2}}\left[-(1-u) p U d t^{2}+\frac{d u^{2}}{(1-u) p U}+V d x^{2}+V d y^{2}\right]$,

$A=\mu(1-u) a d t$,

$\Phi=u^{3-\Delta} \phi$

where $p(u)=1+u+u^{2}-\mu^{2} u^{3} / 4$ and $\mu$ is the chemical potential of the dual boundary field theory. $U, V, a$ and $\phi$ are the function of the radial coordinate $u$ only. Beyond any details of EOMs, the asymptotic behavior of $\phi(u)$ at infinity follows

$\phi(u)=\phi_{0}+\phi_{1} u^{2 \Delta-3}$, where $\phi_{0}$ is identified as the source, which corresponds to the coupling of the boundary QFT and deforms it, and $\phi_{1}$ as the expectation. For the given scalar field mass $m^{2}$ and the coupling parameter $\alpha$, each black brane solution is specified by two scaling-invariant parameters: the Hawking temperature $T \equiv \hat{T} / \mu$ with $\hat{T}=\left(12-\mu^{2}\right) U(1) / 16 \pi$ and the coupling $\lambda \equiv \phi_{0} / \mu^{3-\Delta}$. Without loss of generality, we set $a(0)=1$. In this paper, to more clearly see the effect from the Yukawa coupling, we would like to turn off $\phi_{0}$, i.e., setting $\phi_{0}=0$. It shall be surely interesting to further study the effect of the latter coupling and we leave for the future study.

At the UV boundary, $u \rightarrow 0$, we demand that the geometry approaches $A d S_{4}$ with deformations corresponding to chemical potential $\mu$ and $\phi$ follows the behavior of Eq. (6). At the horizon, $u \rightarrow 1$, we impose the regular boundary conditions. This demand leads to an expansion at the horizon as

$$
\begin{aligned}
U & =4 \pi T(1-u)+\ldots, \\
V & =V_{0}+V_{1}(1-u)+\ldots, \\
a & =a_{0}(1-u)+a_{1}(1-u)^{2}+\ldots, \\
\phi & =\bar{\phi}_{0}+\bar{\phi}_{1}(1-u)+\ldots
\end{aligned}
$$

There are four independent constants $V_{0}, V_{1}, a_{0}$ and $\bar{\phi}_{0}$ in the above expansion. Then we can numerically solve the EOM (3). We show the profile of scalar field for sample $\alpha$ and temperature $T$ in Fig. 1. From the figure, we see that the value of $\Phi(u)$ at the horizon increases as $\alpha$ increases for fixed temperature $T$. While for fixed $\alpha$, the value of $\Phi(u)$ at the horizon increases as the temperature decreases. Here we only focus on the case of $\Delta=2$, for which the scalar operator is relevant one. For other value of $\Delta$, we expect the similar results and shall discuss them in future.

\section{Dirac equation}

We consider the following Dirac action with Yukawa interactions between the spinor field and the scalar field over this gravitational background

$$
\begin{aligned}
& S_{D}=i \int d^{4} x \sqrt{-g} \bar{\zeta}\left(\Gamma^{a} \mathcal{D}_{a}-m_{\zeta}\right) \zeta, \\
& S_{Y}=\int d^{4} x \sqrt{-g}\left[\eta_{1} \bar{\zeta} \Phi \zeta+\eta_{2} \bar{\zeta} \Phi \Gamma^{5} \zeta+\text { h.c. }\right],
\end{aligned}
$$

where $\Gamma^{a}=\left(e_{\mu}\right)^{a} \Gamma^{\mu}$ and $\mathcal{D}_{a}=\partial_{a}+\frac{1}{4}\left(\omega_{\mu \nu}\right)_{a} \Gamma^{\mu \nu}-i q A_{a}$ with $\left(e_{\mu}\right)^{a}$ and $\left(\omega_{\mu \nu}\right)_{a}$ being a set of orthogonal normal vector bases and the spin connection 1 -forms, respectively. $\Gamma^{5}$ is the chirality matrix satisfying $\left\{\Gamma^{5}, \Gamma^{\mu}\right\}=0$. The action (8a) is stimulated by [39-41] to study the fermionic response in holographic framework. And then, lots of extending studies over more general background have been widely explored, 
Fig. 1 Plots of $\Phi(u)$ as the function of $u$ for different $\alpha$ and different $T$. Left plot is for $T=0.01$ and right plot is for $\alpha=5$

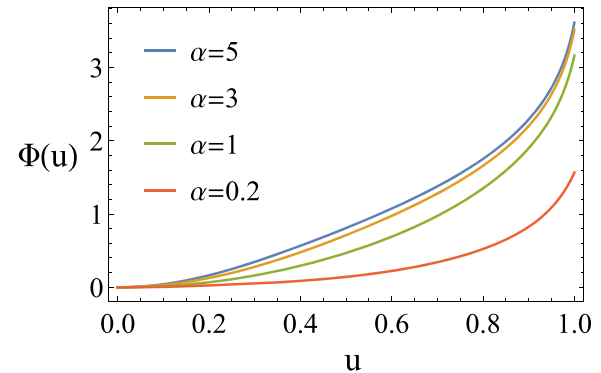

see [42-54] and therein. Here, we introduce the novel terms, Yukawa coupling terms, including the usual scalar Yukawa coupling and the pseudoscalar Yukawa coupling, in (8b).

The Dirac equation can be deduced from the above actions (8a) and (8b)

$\Gamma^{a} \mathcal{D}_{a} \zeta-m_{\zeta} \zeta-i \eta_{1} \Phi \zeta-i \eta_{2} \Phi \Gamma^{5} \zeta=0$.

To cancel off the spin connection, we can make a redefinition of $\zeta=\left(g_{t t} g_{x x} g_{y y}\right)^{-\frac{1}{4}} \mathcal{F}$. At the same time, by the Fourier expansion with $k_{x}=k$ and $k_{y}=0$,

$\mathcal{F}=\int \frac{d \omega d k}{2 \pi} F(u, k) e^{-i \omega t+i k x}$,

one has

$$
\begin{aligned}
& \frac{1}{\sqrt{g_{u u}}} \Gamma^{3} \partial_{u} F-\frac{1}{\sqrt{g_{t t}}} \Gamma^{0}\left(i \omega+i q A_{t}\right) F \\
& -\frac{1}{\sqrt{g_{x x}}} \Gamma^{1} i k F+m_{\zeta} F+i \eta_{1} \Phi F+i \eta_{2} \Phi \Gamma^{5} F=0 .
\end{aligned}
$$

Choose the following gamma matrices

$$
\begin{gathered}
\Gamma^{3}=\left(\begin{array}{cc}
-\sigma^{3} & 0 \\
0 & -\sigma^{3}
\end{array}\right), \quad \Gamma^{0}=\left(\begin{array}{cc}
i \sigma^{1} & 0 \\
0 & i \sigma^{1}
\end{array}\right), \\
\Gamma^{1}=\left(\begin{array}{cc}
-\sigma^{2} & 0 \\
0 & \sigma^{2}
\end{array}\right), \\
\Gamma^{2}=\left(\begin{array}{cc}
0 & \sigma^{2} \\
\sigma^{2} & 0
\end{array}\right), \quad \Gamma^{5}=\left(\begin{array}{cc}
0 & i \sigma^{2} \\
-i \sigma^{2} & 0
\end{array}\right),
\end{gathered}
$$

and split the 4-component spinor into two 2-component spinors as $F=\left(F_{1}, F_{2}\right)^{T}$, one has

$$
\begin{aligned}
& \frac{1}{\sqrt{g_{u u}}} \partial_{u}\left(\begin{array}{c}
F_{1}(k) \\
F_{2}(k)
\end{array}\right)-m_{\zeta} \sigma^{3} \otimes\left(\begin{array}{c}
F_{1}(k) \\
F_{2}(k)
\end{array}\right) \\
& \quad+\left(\omega+q A_{t}\right) \frac{1}{\sqrt{g_{t t}}} i \sigma^{2} \otimes\left(\begin{array}{c}
F_{1}(k) \\
F_{2}(k)
\end{array}\right) \\
& \mp k \frac{1}{\sqrt{g_{x x}}} \sigma^{1} \otimes\left(\begin{array}{c}
F_{1}(k) \\
F_{2}(k)
\end{array}\right) \\
& \quad-i \eta_{1} \Phi \sigma^{3} \otimes\left(\begin{array}{c}
F_{1}(k) \\
F_{2}(k)
\end{array}\right) \pm i \eta_{2} \Phi \sigma^{1} \otimes\left(\begin{array}{l}
F_{2}(k) \\
F_{1}(k)
\end{array}\right)=0 .
\end{aligned}
$$

Furthermore, by the decomposition $F_{\alpha} \equiv\left(\mathcal{A}_{\alpha}, \mathcal{B}_{\alpha}\right)^{T}$ with $\alpha=1,2$, the above Dirac equation can be expressed as

$$
\begin{gathered}
\left(\frac{1}{\sqrt{g_{u u}}} \partial_{u} \mp m_{\zeta}\right)\left(\begin{array}{c}
\mathcal{A}_{1} \\
\mathcal{B}_{1}
\end{array}\right) \pm\left(\omega+q A_{t}\right) \frac{1}{\sqrt{g_{t t}}}\left(\begin{array}{c}
\mathcal{B}_{1} \\
\mathcal{A}_{1}
\end{array}\right) \\
-\frac{k}{\sqrt{g_{x x}}}\left(\begin{array}{c}
\mathcal{B}_{1} \\
\mathcal{A}_{1}
\end{array}\right) \mp i \eta_{1} \Phi\left(\begin{array}{c}
\mathcal{A}_{1} \\
\mathcal{B}_{1}
\end{array}\right)+i \eta_{2} \Phi\left(\begin{array}{c}
\mathcal{B}_{2} \\
\mathcal{A}_{2}
\end{array}\right)=0 \\
\left(\frac{1}{\sqrt{g_{u u}}} \partial_{u} \mp m_{\zeta}\right)\left(\begin{array}{c}
\mathcal{A}_{2} \\
\mathcal{B}_{2}
\end{array}\right) \pm\left(\omega+q A_{t}\right) \frac{1}{\sqrt{g_{t t}}}\left(\begin{array}{c}
\mathcal{B}_{2} \\
\mathcal{A}_{2}
\end{array}\right) \\
+\frac{k}{\sqrt{g_{x x}}}\left(\begin{array}{c}
\mathcal{B}_{2} \\
\mathcal{A}_{2}
\end{array}\right) \mp i \eta_{1} \Phi\left(\begin{array}{c}
\mathcal{A}_{2} \\
\mathcal{B}_{2}
\end{array}\right)-i \eta_{2} \Phi\left(\begin{array}{c}
\mathcal{B}_{1} \\
\mathcal{A}_{1}
\end{array}\right)=0 .
\end{gathered}
$$

At the horizon, we can find that

$$
\partial_{u}\left(\begin{array}{c}
\mathcal{A}_{\alpha}(u, \mathbf{k}) \\
\mathcal{B}_{\alpha}(u, \mathbf{k})
\end{array}\right) \pm \frac{\omega}{4 \pi T} \frac{1}{1-u}\left(\begin{array}{c}
\mathcal{B}_{\alpha}(u, \mathbf{k}) \\
\mathcal{A}_{\alpha}(u, \mathbf{k})
\end{array}\right)=0
$$

In order to obtain the retarded Green function on the boundary by holography, the independent ingoing boundary condition should be imposed at the horizon, i.e.,

$$
\left(\begin{array}{c}
\mathcal{A}_{\alpha}(u, \mathbf{k}) \\
\mathcal{B}_{\alpha}(u, \mathbf{k})
\end{array}\right)=c_{\alpha}\left(\begin{array}{c}
1 \\
-i
\end{array}\right)(1-u)^{-\frac{i \omega}{4 \pi T}}
$$

Near the AdS boundary, the Dirac field reduces to

$$
\left(\begin{array}{l}
\mathcal{A}_{\alpha} \\
\mathcal{B}_{\alpha}
\end{array}\right) \approx a_{\alpha} u^{m_{\zeta}}\left(\begin{array}{l}
1 \\
0
\end{array}\right)+b_{\alpha} u^{-m_{\zeta}}\left(\begin{array}{l}
0 \\
1
\end{array}\right) .
$$

And so by holography, the retarded Green function can be read off

$a_{\alpha}=G_{\alpha \alpha^{\prime}} b_{\alpha^{\prime}}$.

Note that since the four components of the Dirac fields couple to one another, we need to construct a basis of finite solutions, $\left(\mathcal{A}_{\alpha}^{I}, \mathcal{B}_{\alpha}^{I}\right)$ and $\left(\mathcal{A}_{\alpha}^{I I}, \mathcal{B}_{\alpha}^{I I}\right)$, to obtain the boundary Green function. We are mainly interested in the measurable spectral function, which is $A\left(\omega, k_{x}, k_{y}\right) \sim \operatorname{Im}(\operatorname{Tr} G)$. In the next section, we shall numerically study the fermionic spectral function and explore its properties from Yukawa coupling. 

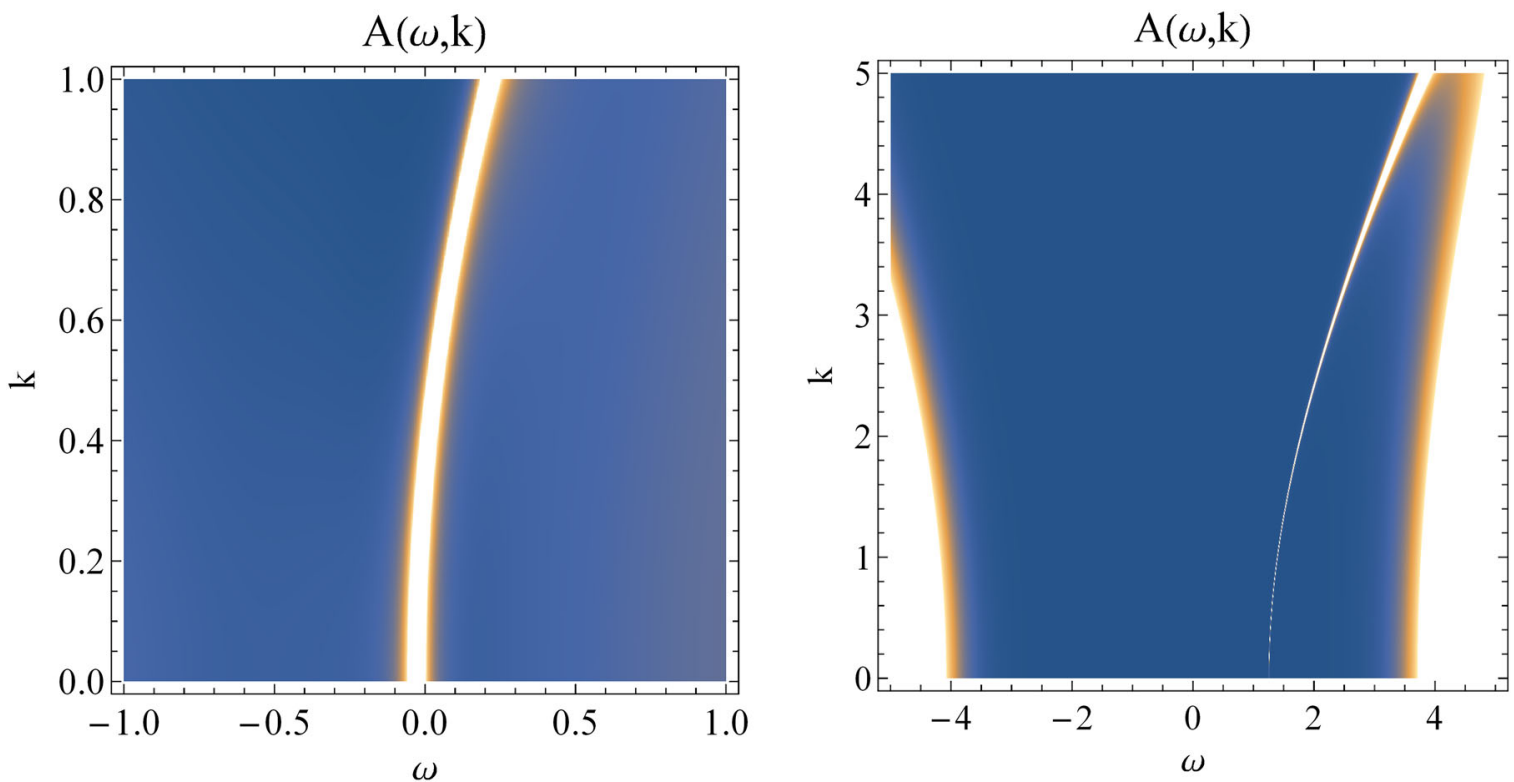

Fig. 2 Density plots of spectral function $A(\omega, k)$ for $\alpha=5$ and $\Delta=2$ at $T=0.01$. Left plot is for $\eta_{2}=0.5$ and right plot for $\eta_{2}=4$

Fig. 3 Spectral function $A(\omega, k)$ for sample $k$ with $\alpha=5$ and $\Delta=2$ at $T=0.01$. Left plot is for $\eta_{2}=0.5$ and right plot for $\eta_{2}=4$
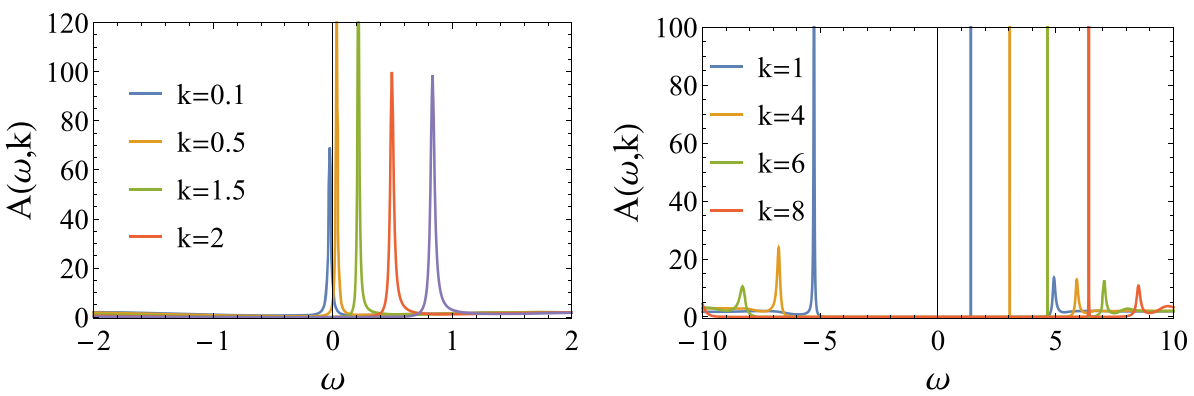

\section{Emergence of the dynamical gap}

The formation of the dynamical gap is the topic of this paper. But the preliminary exploration indicates that the Yukawa coupling $\eta_{1}$ cannot drive the formation of the gap (see "Appendix A"). Therefore, we shall only turn on the pseudoscalar Yukawa coupling term $\eta_{2}$ in what follows.

For definiteness, through this paper, we shall set $m_{\zeta}=0$. We first study the fermionic spectrum at fixed temperature $T=0.01$. By numerically solving the Dirac Eqs. (14) and (15), we show the density plots of spectral functions $A(\omega, k)$ with $\eta_{2}=0.5$ and $\eta_{2}=4$ in Fig. 2 . The quasi-particle-like excitation is observed at $\omega=0$ in the spectrum for small $\eta_{2}$, for example, $\eta_{2}=0.5$ (left plot in Fig. 2), which is similar to the case of $\eta_{2}=0$, i.e., RN-AdS background [40]. With the increase of the pseudoscalar Yukawa coupling strength and beyond some critical value, a dynamical gap opens at $\omega=0$ in the spectrum (see right plot in Fig. 2). In Fig. 3, we also show $A(\omega, k)$ with $\eta_{2}=0.5$ and $\eta_{2}=4$ for sample values of $k$. For $\eta_{2}=0.5$, some sharp peaks distribute near $\omega=0$, which can be identified with

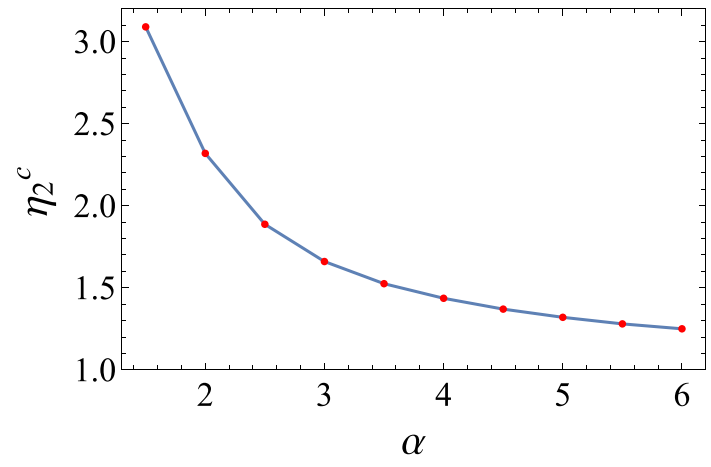

Fig. 4 The relation between $\alpha$ and $\eta_{2}^{c}$ for fixed $T=0.01$

the quasi-particle-like excitation. But for large $\eta_{2}$, the peak disappears around $\omega=0$ and a gap opens. Especially, we observe that the peak are pushed away from $\omega=0$ as $k$ becomes larger. This result indicates that the gap exists for all $k{ }^{1}$

1 The robustness of the formation of the gap is further confirmed in the density of state (DOS) of the spectral function in what follows. 
Table $1 \eta_{2}^{c}$ with different $\alpha$ for $T=0.01$

\begin{tabular}{lllllll}
\hline$\alpha$ & 1.5 & 2 & 3 & 4 & 5 & 6 \\
\hline$\eta_{2}^{c}$ & 3.090 & 2.319 & 1.660 & 1.436 & 1.320 & 1.250 \\
\hline
\end{tabular}

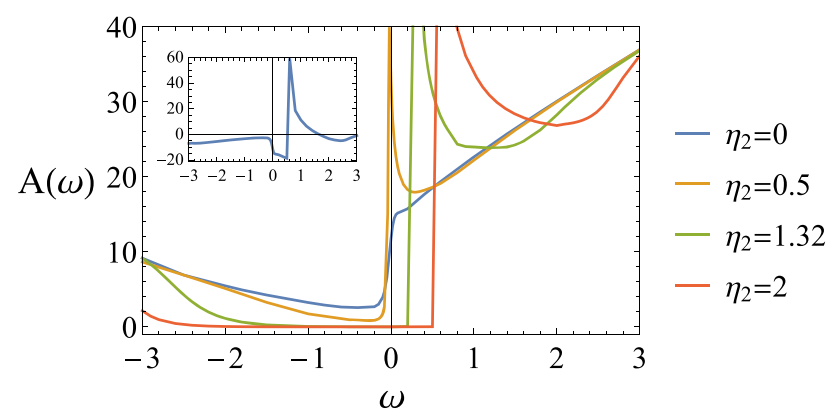

Fig. $5 A(\omega)$ for different $\eta_{2}$. Here, we have set $\alpha=5$ and $T=0.01$. The inset shows $A\left(\omega, \eta_{2}=2\right)-A\left(\omega, \eta_{2}=0\right)$ as a function of $\omega$

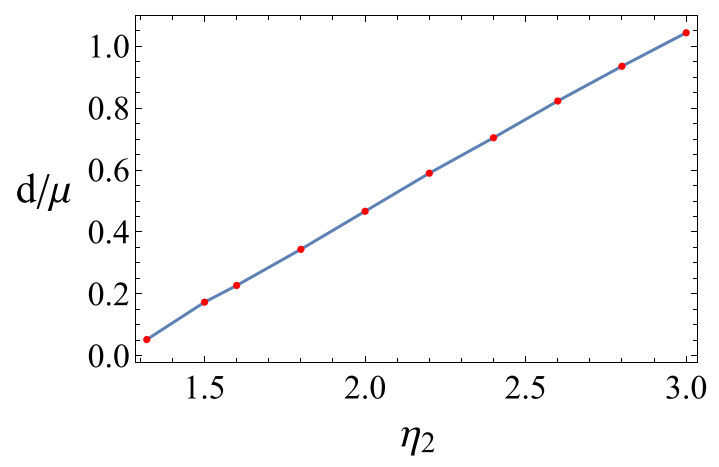

Fig. 6 The relation between $\eta_{2}$ and the width of the gap $d / \mu$ for fixed $\alpha=5$ and $T=0.01$
To gain a quantitative understanding of the spectral measure with Yukawa coupling, we study the density of state (DOS) $A(\omega)$, which signals the total weight of the spectral measure and is defined by the integral of $A(\omega, k)$ over $k$. In terms of the DOS, we can introduce the critical value of the onset of the gap $\eta_{2}^{c}$, which is identified as that the DOS at the Fermi level $(\omega=0)$ drops below some small number (here, we take $10^{-3}$ in numerical calculations). The critical value $\eta_{2}^{c}$ can signal the formation of the gap. Figure 4 and Table 1 show the relation between $\alpha$ and $\eta_{2}^{c}$ for fixed $T=0.01$. We see that with the increase of $\alpha$, the $\eta_{2}^{c}$ decreases. It indicates that the gap opens easier for larger $\alpha$. It is because the low frequency spectrum probes the near horizon geometry. While for larger $\alpha$, the amplitude of the scalar field near the horizon is more evident (see left plot in Fig. 1).

Now, we turn to study the spectrum on all energy scales. Figure 5 shows the DOS $A(\omega)$ for different $\eta_{2}$. First, this figure again clearly exhibits that the formation of the gap is robust for all $k$ because the integral is done over all $k$. Second, at low frequency region (the absolute value $|\omega|$ is small), the Yukawa coupling results in rearrangements of the spectrum. With the increase of the Yukawa coupling $\eta_{2}$, the spectral weight transfers from the low energy band $(\omega<0)$ to the high energy band $(\omega>0)$. But at the high frequency region (the absolute value $|\omega|$ is large), the DOS for all $\eta_{2}$ tend to the same value. Therefore, the transfer of spectral weight is only over low energy scales but not all energy scales. It is different from the Mott physics, for which the transfer of spectral weight is over all energy scales. The reason is that the scalar field has a non-trivial profile near the horizon but vanishes on the conformal boundary (see Fig. 1).
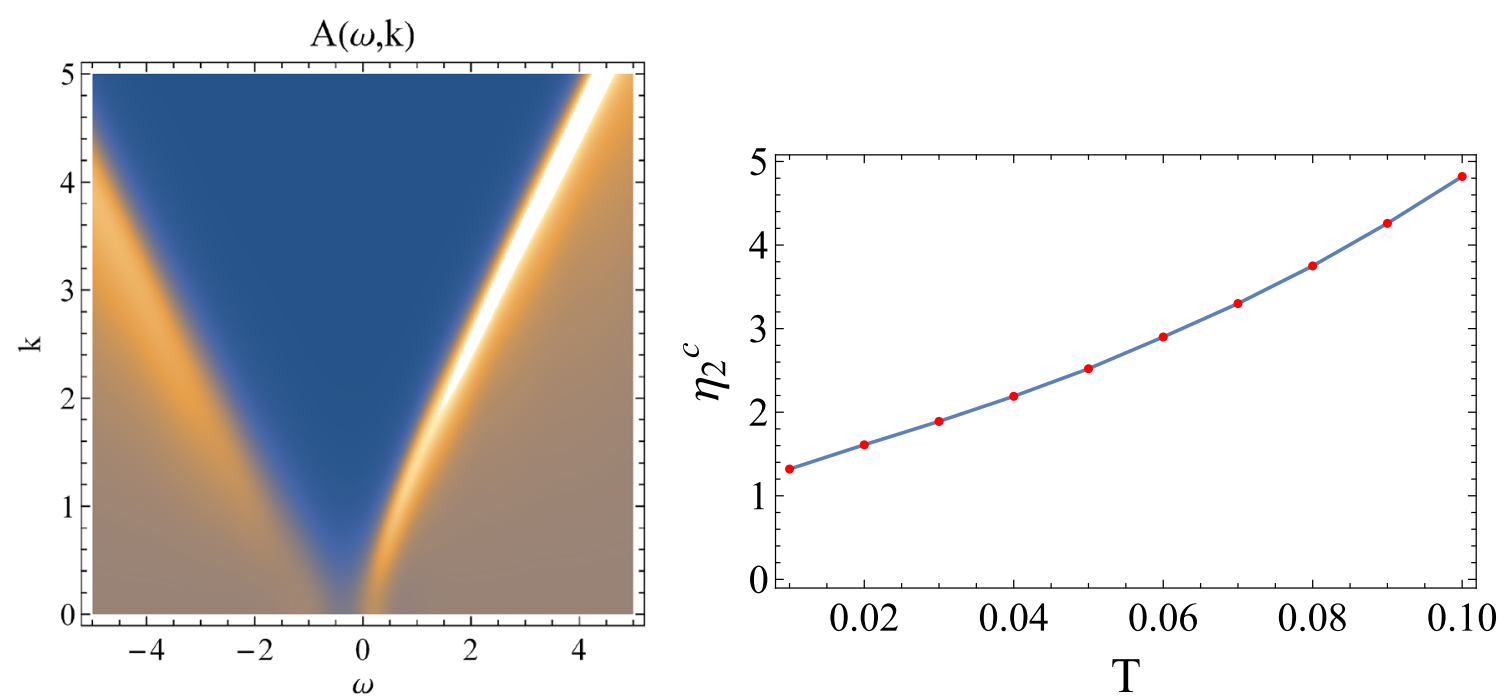

Fig. 7 Left plot: the density plot of spectral function $A(\omega, k)$ for $\alpha=5$ and $\eta_{2}=4$ at $T=0.22$. Right plot: the relation between $T$ and $\eta_{2}^{c}$ for fixed $\alpha=5$ 
Another characteristic quantity is the width of the gap $d / \mu$, which is shown in Fig. 6 for fixed $\alpha=5$ and $T=0.01$. We see that the width of the gap $d / \mu$ linearly increases as the Yukawa coupling $\eta_{2}$. It confirms again that the Yukawa coupling drives the formation of the gap.

Next, we study the evolution of the spectral function with temperature. For the dipole coupling fermionic system, it has been shown that as the temperature increases, the gap gradually closes. Here, we also find that the temperature plays a similar role for the Yukawa coupling fermionic system. Left plot in Fig. 7 shows the density plot of spectral function $A(\omega, k)$ for $\alpha=5$ and $\eta_{2}=4$ at $T=0.22$. Obviously, the gap closes at this moment. We also quantitatively gives the relation between $T$ and $\eta_{2}^{c}$ for fixed $\alpha=5$, which is shown in the right plot in Fig. 7. As the temperature $T$ increases, the critical value of the onset of the gap $\eta_{2}^{c}$ increases.

\section{Conclusion and discussion}

In this paper, we introduce a novel coupling term, Yukawa interaction between the scalar field and the spinor field, in the Dirac action and study its fermionic response. We observe that a gap dynamically emerges. Moreover, the Yukawa coupling results in rearrangements of the spectrum at low frequency region but the DOS for all $\eta_{2}$ tends to the same value at the high frequency region. It is because the scalar field has a non-trivial profile near the horizon but vanishes on the AdS boundary. This phenomena is different from the Mott physics. We also study the evolution of the spectral function with temperature. We find that with the increase of temperature, the gap closes. More detailed studies deserved deeply exploring such that we can have more understanding for our fermionic system.
Lots of works deserve further studying. One immediate topic is to explore the non-relativistic fermionic spectrum as [55-57] with Yukawa coupling to see the effect of Yukawa coupling on the flat band. Also, we also hope to implement the Fermi arc on our model on top of some anisotropic background, for example, [58].

Acknowledgements This work is supported by the Natural Science Foundation of China under Grant nos. 11775036 and 11847313.

Data Availability Statement This manuscript has no associated data or the data will not be deposited. [Authors' Comment: This paper is a theoretical study, for which no data is deposited.]

Open Access This article is distributed under the terms of the Creative Commons Attribution 4.0 International License (http://creativecomm ons.org/licenses/by/4.0/), which permits unrestricted use, distribution, and reproduction in any medium, provided you give appropriate credit to the original author(s) and the source, provide a link to the Creative Commons license, and indicate if changes were made.

Funded by $\mathrm{SCOAP}^{3}$.

\section{Appendix A: Fermionic spectrum for $\eta_{1} \neq 0$}

In this Appendix, we shall give a brief discussion on the effect of the Yukawa coupling $\eta_{1}$. First, a lot of numerical analysis indicates that the Yukawa coupling $\eta_{1}$ is constrained in a small parameter space because of the requirement of the positive definiteness of the spectral function. Therefore, we shall explore the effect of $\eta_{1}$ in the small $\eta_{1}$ space.

Figure 8 show the density plots of spectral function $A(\omega, k)$ for $\alpha=5, \Delta=2$ and $\eta_{2}=0$ at $T=0.01$. Left plot is for $\eta_{1}=-0.1$ and right plot for $\eta_{1}=0.01 .^{2}$ From this figure, we cannot observe the formation of gap in the allowed parameter space.

\footnotetext{
$\overline{2}$ Because of the requirement of the positive definiteness of the spectral function, $\eta_{1}$ is approximately constrained in the regions of $-0.1 \leq$ $\eta_{1} \leq 0.01$ for $\alpha=5$.
} 

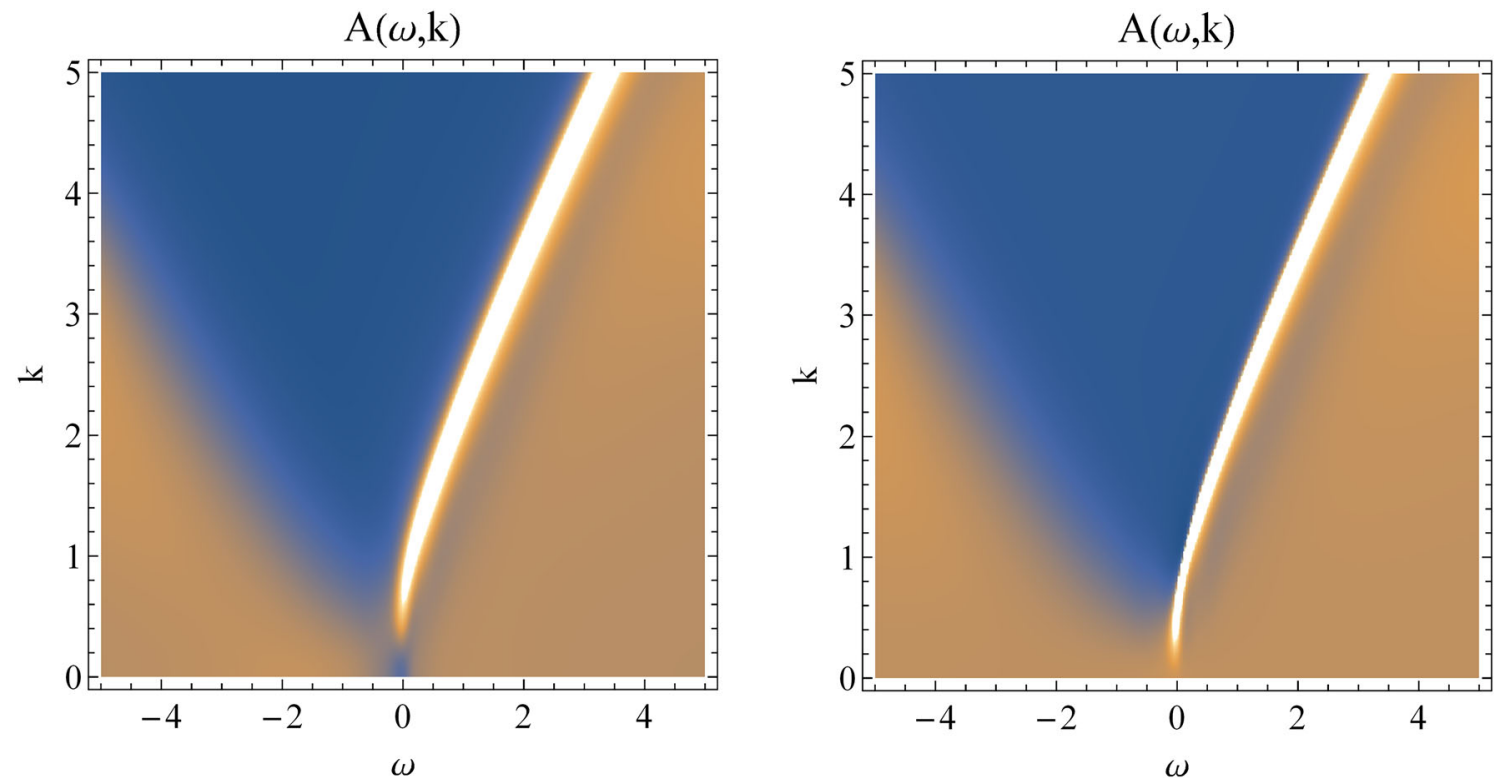

Fig. 8 Density plots of spectral function $A(\omega, k)$ for $\alpha=5, \Delta=2$ and $\eta_{2}=0$ at $T=0.01$. Left plot is for $\eta_{1}=-0.1$ and right plot for $\eta_{1}=0.01$

\section{References}

1. G.'t Hooft, A two-dimensional model for mesons. Nuclear Phys. B 75, 461 (1974)

2. I. Dzyaloshinskii, Some consequences of the Luttinger theorem: The Luttinger surfaces in non-Fermi liquids and Mott insulators. Phys. Rev. B 68, 085113 (2003)

3. J.M. Maldacena, The Large N limit of superconformal field theories and supergravity. Adv. Theor. Math. Phys. 2, 231 (1998). arxiv:hep-th/9711200

4. S.S. Gubser, I.R. Klebanov, A.M. Polyakov, Gauge theory correlators from noncritical string theory. Phys. Lett. B 428, 105 (1998). arxiv:hep-th/9802109

5. E. Witten, Anti-de Sitter space and holography. Adv. Theor. Math. Phys. 2, 253 (1998). arxiv:hep-th/9802150

6. O. Aharony, S.S. Gubser, J.M. Maldacena, H. Ooguri, Y. Oz, Large $\mathrm{N}$ field theories, string theory and gravity. Phys. Rep. 323, 183 (2000). arxiv:hep-th/9905111

7. E. Kiritsis, J. Ren, On holographic insulators and supersolids. JHEP 1509, 168 (2015). arXiv:1503.03481 [hep-th]

8. C. Charmousis, B. Gouteraux, B.S. Kim, E. Kiritsis, R. Meyer, Effective Holographic Theories for low-temperature condensed matter systems. JHEP 1011, 151 (2010). arXiv:1005.4690 [hepth]

9. B. Gouteraux, E. Kiritsis, Generalized holographic quantum criticality at finite density. JHEP 1112, 036 (2011). arXiv:1107.2116 [hep-th]

10. B. Gouteraux, E. Kiritsis, Quantum critical lines in holographic phases with (un)broken symmetry. JHEP 1304, 053 (2013). arXiv:1212.2625 [hep-th]

11. Y. Ling, P. Liu, C. Niu, J.P. Wu, Building a doped Mott system by holography. Phys. Rev. D 92(8), 086003 (2015). arXiv:1507.02514 [hep-th]

12. Y. Ling, P. Liu, J.P. Wu, A novel insulator by holographic Q-lattices. JHEP 1602, 075 (2016). arXiv:1510.05456 [hep-th]

13. M. Baggioli, O. Pujolas, On effective holographic Mott insulators. JHEP 1612, 107 (2016). arXiv:1604.08915 [hep-th]
14. T. Andrade, A. Krikun, K. Schalm, J. Zaanen, Doping the holographic Mott insulator. Nat. Phys. (2018). arXiv:1710.05791 [hepth]

15. M. Edalati, R.G. Leigh, P.W. Phillips, Dynamically generated Mott gap from holography. Phys. Rev. Lett. 106, 091602 (2011). arXiv: 1010.3238 [hep-th]

16. M. Edalati, R.G. Leigh, K.W. Lo, P.W. Phillips, Dynamical gap and cuprate-like physics from holography. Phys. Rev. D 83, 046012 (2011). arXiv:1012.3751 [hep-th]

17. J.P. Wu, H.B. Zeng, Dynamic gap from holographic fermions in charged dilaton black branes. JHEP 1204, 068 (2012). arXiv: 1201.2485 [hep-th]

18. X.M. Kuang, B. Wang, J.P. Wu, Dipole coupling effect of holographic fermion in the background of charged Gauss-Bonnet AdS black hole. JHEP 1207, 125 (2012). arXiv:1205.6674 [hep-th]

19. W.Y. Wen, S.Y. Wu, Dipole Coupling Effect of Holographic Fermion in Charged Dilatonic Gravity. Phys. Lett. B 712, 266 (2012). arXiv:1202.6539 [hep-th]

20. X.M. Kuang, B. Wang, J.P. Wu, Dynamical gap from holography in the charged dilaton black hole. Class. Quantum Gravity 30, 145011 (2013). arXiv:1210.5735 [hep-th]

21. J.P. Wu, Emergence of gap from holographic fermions on charged Lifshitz background. JHEP 1304, 073 (2013)

22. J.P. Wu, The charged Lifshitz black brane geometry and the bulk dipole coupling. Phys. Lett. B 728, 450 (2014)

23. X.M. Kuang, E. Papantonopoulos, B. Wang, J.P. Wu, Dynamically generated gap from holography in the charged black brane with hyperscaling violation. JHEP 1504, 137 (2015). arXiv:1411.5627 [hep-th]

24. Y. Ling, P. Liu, C. Niu, J.P. Wu, Z.Y. Xian, Holographic fermionic system with dipole coupling on Q-lattice. JHEP 1412, 149 (2014). arXiv: 1410.7323 [hep-th]

25. G. Vanacore, P.W. Phillips, Minding the gap in holographic models of interacting fermions. Phys. Rev. D 90, 044022 (2014). arXiv:1405.1041 [cond-mat.str-el]

26. Z. Fan, Dynamic Mott gap from holographic fermions in geometries with hyperscaling violation. JHEP 1308, 119 (2013). arXiv:1305.1151 [hep-th] 
27. L.Q. Fang, X.M. Kuang, B. Wang, J.P. Wu, Fermionic phase transition induced by the effective impurity in holography. JHEP 1511, 134 (2015). arXiv:1507.03121 [hep-th]

28. J.P. Wu, Holographic fermionic spectrum from Born-Infeld AdS black hole. Phys. Lett. B 758, 440 (2016). arXiv:1705.06707 [hepth]

29. X.M. Kuang, J.P. Wu, Effect of quintessence on holographic fermionic spectrum. Eur. Phys. J. C 77(10), 670 (2017)

30. L.Q. Fang, X.M. Kuang, J.P. Wu, The holographic fermions dual to massive gravity. Sci. China Phys. Mech. Astron. 59(10), 100411 (2016)

31. L.Q. Fang, X.H. Ge, X.M. Kuang, Holographic fermions with running chemical potential and dipole coupling. Nucl. Phys. B 877, 807 (2013). arXiv:1304.7431 [hep-th]

32. Y. Ling, P. Liu, C. Niu, J.P. Wu, Pseudo-gap phase and duality in a holographic fermionic system with dipole coupling on Q-lattice. Chin. Phys. C 40(4), 043102 (2016). arXiv:1602.06062 [hep-th]

33. Y. Seo, G. Song, Y.H. Qi, S.J. Sin, Mott transition with holographic spectral function. arXiv:1803.01864 [hep-th]

34. G. Vanacore, S.T. Ramamurthy, P.W. Phillips, Evolution of holographic Fermi arcs from a Mott insulator. arXiv:1508.02390 [condmat.str-el]

35. T. Faulkner, G.T. Horowitz, J. McGreevy, M.M. Roberts, D. Vegh, Photoemission experiments on holographic superconductors. JHEP 1003, 121 (2010). arXiv:0911.3402 [hep-th]

36. D. Vegh, Fermi arcs from holography. arXiv:1007.0246 [hep-th]

37. F. Benini, C.P. Herzog, A. Yarom, Holographic Fermi arcs and a d-wave gap. Phys. Lett. B 701, 626 (2011). arXiv:1006.0731 [hepth]

38. A. Lucas, T. Sierens, W. Witczak-Krempa, Quantum critical response: from conformal perturbation theory to holography. JHEP 1707, 149 (2017). arXiv:1704.05461 [hep-th]

39. S.S. Lee, A non-Fermi liquid from a charged black hole: a critical Fermi ball. Phys. Rev. D 79, 086006 (2009). arXiv:0809.3402 [hepth]

40. H. Liu, J. McGreevy, D. Vegh, Non-Fermi liquids from holography. Phys. Rev. D 83, 065029 (2011). arXiv:0903.2477 [hep-th]

41. T. Faulkner, H. Liu, J. McGreevy, D. Vegh, Emergent quantum criticality, Fermi surfaces, and AdS(2). Phys. Rev. D 83, 125002 (2011). arXiv:0907.2694 [hep-th]

42. J.P. Wu, Holographic fermions in charged Gauss-Bonnet black hole. JHEP 1107, 106 (2011). arXiv:1103.3982 [hep-th]

43. Y. Liu, K. Schalm, Y.W. Sun, J. Zaanen, Lattice potentials and fermions in holographic non Fermi-liquids: hybridizing local quantum criticality. JHEP 1210, 036 (2012). arXiv:1205.5227 [hep-th]

44. Y. Ling, C. Niu, J.P. Wu, Z.Y. Xian, H.B. Zhang, Holographic Fermionic liquid with lattices. JHEP 1307, 045 (2013). arXiv:1304.2128 [hep-th]
45. J.P. Wu, Some properties of the holographic fermions in an extremal charged dilatonic black hole. Phys. Rev. D 84, 064008 (2011). arXiv:1108.6134 [hep-th]

46. U. Gursoy, E. Plauschinn, H. Stoof, S. Vandoren, Holography and ARPES sum-rules. JHEP 1205, 018 (2012). arXiv:1112.5074 [hepth]

47. M. Alishahiha, M.R. Mohammadi Mozaffar, A. Mollabashi, Fermions on Lifshitz background. Phys. Rev. D 86, 026002 (2012). arXiv:1201.1764 [hep-th]

48. L.Q. Fang, X.H. Ge, X.M. Kuang, Holographic fermions in charged Lifshitz theory. Phys. Rev. D 86, 105037 (2012). arXiv:1201.3832 [hep-th]

49. W.J. Li, J.P. Wu, Holographic fermions in charged dilaton black branes. Nucl. Phys. B 867, 810 (2013). arXiv:1203.0674 [hep-th]

50. J. Wang, Schrodinger Fermi liquids. Phys. Rev. D 89(4), 046008 (2014). arXiv:1301.1986 [hep-th]

51. J.P. Wu, Holographic fermions on a charged Lifshitz background from Einstein-Dilaton-Maxwell model. JHEP 1303, 083 (2013)

52. X.M. Kuang, E. Papantonopoulos, B. Wang, J.P. Wu, Formation of Fermi surfaces and the appearance of liquid phases in holographic theories with hyperscaling violation. JHEP 1411, 086 (2014). arXiv:1409.2945 [hep-th]

53. L.Q. Fang, X.H. Ge, J.P. Wu, H.Q. Leng, Anisotropic Fermi surface from holography. Phys. Rev. D 91(12), 126009 (2015). arXiv:1409.6062 [hep-th]

54. L.Q. Fang, X.M. Kuang, Holographic Fermions in anisotropic Einstein-Maxwell-Dilaton-Axion theory. Adv. High Energy Phys. 2015, 658607 (2015)

55. J.N. Laia, D. Tong, A holographic flat band. JHEP 1111, 125 (2011). arXiv:1108.1381 [hep-th]

56. W.J. Li, H.B. Zhang, Holographic non-relativistic fermionic fixed point and bulk dipole coupling. JHEP 1111, 018 (2011). arXiv: 1110.4559 [hep-th]

57. W.J. Li, R. Meyer, H.B. Zhang, Holographic non-relativistic fermionic fixed point by the charged dilatonic black hole. JHEP 1201, 153 (2012). arXiv:1111.3783 [hep-th]

58. N. Iizuka, K. Maeda, Study of anisotropic black branes in asymptotically anti-de sitter. JHEP 1207, 129 (2012). arXiv:1204.3008 [hep-th]

59. G.B. Arfken, H.J. Weber, Mathematical methods for physicists. A Harcourt Science and Technology Company (2001)

60. C.M. Bender, S.A. Orszag, Advanced Mathematical Methods for Scientists and Engineers (McGraw-Hill, New York, 1978) 